\title{
Detection of Wood Boring Insects' Larvae Based on the Acoustic Signal Analysis and the Artificial Intelligence Algorithm
}

\author{
Piotr BILSKI ${ }^{(1)}$, Piotr BOBIŃSKI ${ }^{(1)}$, Adam KRAJEWSKI ${ }^{(2)}$, Piotr WITOMSKI ${ }^{(2)}$ \\ (1) Institute of Radioelectronics and Multimedia Technology \\ Faculty of Electronics and Information Technology \\ Warsaw University of Technology \\ Nowowiejska 15/19, 00-665 Warsaw, Poland; e-mail: \{pbilski, P.Bobinski\}@ire.pw.edu.pl \\ ${ }^{(2)}$ Department of Wood Science and Wood Protection \\ Warsaw University of Life Sciences \\ Nowoursynowska 166, 02-776 Warsaw, Poland; e-mail: \{adam_krajewski, piotr_witomski\}@sggw.pl
}

(received April 18, 2016; accepted October 21, 2016)

The paper presents an application of signal processing and computational intelligence methods to detect presence of the wood boring insects larvae in the wooden constructions (such as the furniture of buildings). Such insects are one of the main sources of the degradation in such objects, therefore they should be detected as quickly as possible, before inflicting serious damage. The presented work involved the acoustic monitoring for detecting the presence of the larvae inside pieces of wood. An accelerometer was used to record the sound, further analyzed by a computer algorithm extracting features important for artificial-intelligence (AI) based classification employed to detect the old house borer's (Hylotrupes bajulus L.) activity. The presented task is difficult, as the sounds made by the larvae are of relatively low amplitude and the background noise caused by people, electrical appliances or other sources may significantly degrade the accuracy of detection. The classification of sounds is needed to separate sources of noise which deteriorate the proper larva detection and should be suppressed if possible. The employed classification was based on features defined in the time domain followed by the support vector machine used as the binary classifier. The results allowed us to assess the effectiveness of the old house borer's detection by the acoustic analysis enhanced with the AI algorithm.

Keywords: wood boring insects identification; artificial intelligence classification; accelerometer.

\section{Introduction}

Monitoring of the wooden constructions and structures (such as old buildings or furniture) is the important operation executed by the specialists responsible for maintaining them in as good shape as possible. This process is economically justified as such objects are usually highly valuable and have to be protected from the environmental degradation. The wood must be conserved and protected from the decay. The woodworm vermin are responsible for substantial losses and make the task for the renovators difficult. The larvae of the particular beetles spend most of their lives in the pieces of wood, where they are safe and able to feed. Unfortunately, it is difficult to detect their presence in the wooden construction in early stages of infesta- tion, when they are scarce and small. After growing up and breeding, they are easier to identify, but at that stage the damage is already substantial. This makes the early detection crucial and inspires multiple approaches to do the task. The particular insect' specimens feed on different types of wood, which requires individual approach for every species. This forms the demanding practical and research task, requiring development of both signal processing and automated classification methods.

To decrease the damage inflicted to the material, only the non-intrusive approaches are proposed, where the wooden structure remains intact. All methods implemented so far use either visual examination or radiation at the predefined frequency range. Contrary to the $x$-ray and infrared applied before with mixed re- 
sults, the Acoustic Emission (AE) analysis of the feeding larva is the novel approach, drawing attention of researchers. Multiple problems have to be solved in this scheme: the existence of the additive noise, various sound patterns made by the larvae of various age and sizes. Also, the fabric of the wood may be different, depending on its condition, moisture, etc.

The paper presents the novel approach for the old house borer presence detection based on the acoustic analysis of the sound made by larva during the feeding. Its topic and proposed solution is similar to the work presented in (ScHOFIELD, 2011), where time domain features are considered to identify the larvae presence in the wood. The main differences from that work include the analyzed species, the algorithm for distinguishing larva-sounds from the background noise and the insect classification method. Our solutions are simpler while maintaining the high accuracy. We applied the Support Vector Machine (SVM) to automatically identify the presence of the insect in the wood. The large sound data sets (containing almost 200 samples) were used to verify effectiveness of our method. The paper has the following structure. In Sec. 2 the characteristics of the problem of non-intrusive monitoring the larva is introduced. Section 3 presents the approach proposed by Authors. In Sec. 4 the AI-based identification and its requirements are introduced. Section 5 presents the applied SVM binary classifier. Experimental setup and results are discussed in Sec. 6, while the conclusions and future prospects of the method are presented in Sec. 7 .

\section{Woodworm lifecycle characteristics}

The wood boring insects are currently the main cause of damage in the wooden constructions. This is especially true for the portable pieces of art (such as sculptures), rooftops or bearing pillars in the apartments. The most dangerous species of insects destroy the wood by dwelling inside it for many generations, causing its total destruction. The most dangerous insects are old house borer (Hylotrupes bajulus L.) Fig. 1, and common furniture beetle (Anobium punc-

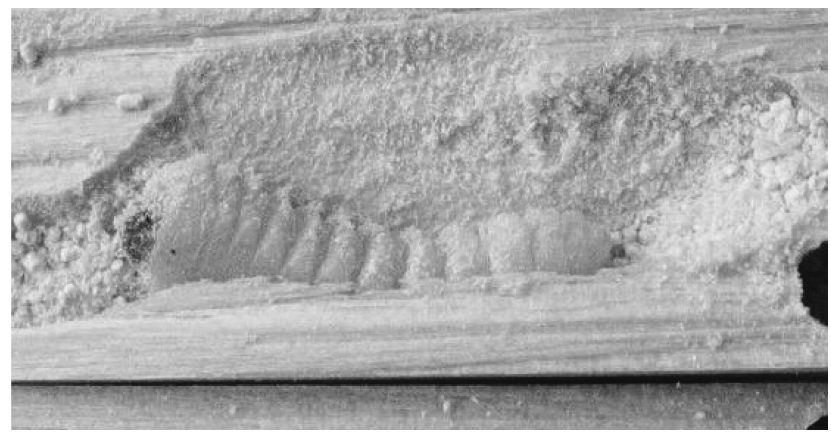

Fig. 1. Old house borer larva. tatum De Geer). Both species exist in the wide areas of Poland, dwelling in various types of trees, which facilitates their aggressive expansion. They are often spotted in so-called skeletal constructions, common in buildings raised in the beginnings of the XXth century. Other species of wood-dwelling insects, such as termites, cause damages inside buildings, feeding on paneling, floor or furniture.

The analysis of the presented insects (especially exploiting the acoustic signals) is influenced by multiple factors. The most important is the temperature, as the larvae activity is high only within the specific range, which should be maintained during the experiments. Although the common furniture beetle and the death watch beetle (Xestobium rufovillosum De Geer) prefer medium temperatures and high humidity (therefore living in the vicinity of basements), the old house borer can be met in the higher temperature locations (common in roofs and attics). Secondly, the size of the specimen influences the characteristics (amplitude and duration) of the generated sound. It was proven in (HETzRONI et al., 2016) that each insect behaves differently and generates specific sounds, depending on both the thickness of wood and structure of the feeding apparatus (CHIAPPINI, NiCOLIALDini, 2011).

The methods of the wood state analysis used so far allow for detecting the existence of the vermin in the relatively late stage of the infestation, i.e. when the damage is visible by the naked eye and therefore difficult to repair. Especially important are the nonintrusive approaches, leading to the quick and cheap assessment of the wood state. The detailed costs of the specific approach depend on the physical phenomenon applied to the task. One of the most important drawbacks of the currently applied approaches is the limited ability of the in-situ implementation. The measurement equipment should be relocated outside the laboratory whenever there is the need to verify the state of the construction or the sculpture in the location far from the research facility. Most of the currently available approaches are used only in the laboratory conditions, which raises questions about their versatility and flexibility:

a) Visual examination of the analyzed object by the human expert. He/she is responsible for finding traces of the insects' existence in the wood (such as holes or feces). Because larvae make sounds during the feeding on the wood, they should be heard as well.

b) Usage of the chemo- and olfactory sensing technique delivered by sniffer dogs. They are able to detect insects during at least the medium stage of infestation. This method requires especially trained dogs and is relatively costly.

c) Measurement of the oxygen consumption by insects. When feeding or moving, the larvae breathe 
more intensely, which can be detected, assuming that the reference level of the gas in the air is known (STUSEK et al., 2000).

d) Application of the videoendoscopy to discover the actual state of the wooden construction. The obtained image allows for identifying the damages made by the wood boring insects (KRAJEWSKI, Witomski, 2005). The disadvantage of this approach is its invasiveness, i.e. the need to insert the device to the interior of the wood.

e) The radiological methods exploiting the Roentgen or neutron radiation. This is the popular approach, because of its high accuracy during the material state assessment. In (OsTERLOH, Nusser, 2014) the approach was applied to the analysis of the antique wooden sculptures, while in (FIALA et al., 2014) the X-rays were used to detect wood boring insects in the boards of the building floor and roof. The supplementary method to the radiological analysis was the electroacoustic wave. Its scope was limited, though, the objective was just to detect the existence of the larvae, not determine their species (as it is proposed in this project).

f) The computer tomography is the extension of the radiological approach. As the traditional method allows for creating the two-dimensional image of the object exposed to the X-rays, the application of the tomography leads to three-dimensional images with high resolution. In (MiKOŁAJSKA et al., 2012) the method was used to create images of the antique sculpture in the plane and space. This enabled localization of the holes created by the feeding larvae. In (STIRR, WADDLE, 1999) tomography images were applied to analyze the condition of the wooden violin.

g) The infrared spectrum allows for detecting the local changes in the temperature. The method is implementable with the help of various camera types. In (HofFmAnN et al., 2013) the application of such a system to analyze the state of the young trees in the forest was described. The method is not of much use in the in-situ investigation, as the sick trees were difficult to distinguish from the healthy ones.

The described methods are characterized by the following flaws, which justify proposing new methodologies:

a) Weak portability, as the hardware used for the analysis is usually large, making it usable mainly in the laboratory (which is especially true for the Roentgen rays and the computer tomography).

b) Methods exploiting the radiation beyond the visible range are useful in visualizing the current state of the wood, but are not accurate enough to detect and identify the living wood boring insects. c) The applied hardware is usually expensive, making it economically profitable only when used intensively in multiple analyses.

d) The analysis of the collected material requires the presence of the human expert. The modern computers are advanced enough to be used for the automated classification of objects based on the vectors of features, describing the particular piece of wood (and the worm inside).

Most of the disadvantages are avoided by the AE analysis. This approach has been tested for the last seventy years, using various types of equipment. The latter started from the standard microphones (PENCE et al., 1954), which were quickly abandoned in favour of piezo-electric or bi-morph sensors (FARR, 2007) or accelerometers (MANkIN et al., 2004). They are currently preferred as they have a higher sensitivity to the sound carried in the solid material such as wood. The ultrasound inspection was also tested (FLEMING et al., 2005) but with poor results. On the other hand, the laser vibrometry (Zorović, CokL, 2015) is the non-contact method with acceptable accuracy. Its disadvantage is the high cost. The considered objects included trees, wooden furniture and pallets for commodity goods. The research conducted using AE covered termites (LEMASTER et al., 1997) and the most dangerous species of Cerambycidae. The method was also considered for old house borer, but its applications were limited to determining the ability of recording the acoustic effects related with the existence and feeding of larvae in the wood (KERNER et al., 1980). It was proven that this method can be used to assess the intensity of infestation of the wooden construction element (KRAJEWSKI et al., 2012). The main problems reported from the above experiments include the difficulty of detecting the larvae presence with the background noise and distinguishing between various species of insects. Unfortunately, both aims are difficult to achieve and so far relied on the intuition and experience of researchers. The automated approaches would be more effective, but they are not fully exploited. Machine learning modules used for the research mainly include Artificial Neural Networks (ANN) (SchOFIELD, 2011). This calls for additional intelligent approaches.

The proposed methodology has the potential not only for the detection of the particular type of larva, but also distinguishing between various species of insects. The scheme is also economically attractive, requiring the minimal sound acquisition equipment. The Authors made the preliminary tests based on the acoustic analysis of the infested pieces of wood (KRAJEWSKI et al., 2012). However, the analysis exploited the human expert manually assessing the state of the object. The intensity of the infestation (the number of larvae and their age) was based on the intuition, therefore it can't be used as the impartial method. 


\section{Non-intrusive wood boring insects detection system}

The proposed system is compliant to the generic architecture of the automated data acquisition (DAQ) and decision making scheme (Fig. 2). Three operations performed here are connected to the main steps of any methodology implemented in the analysis of the actual system. The data acquisition is responsible for collecting the vibrations from the piece of wood with the single accelerometer attached to it using the sticky paste. The DAQ system consists of the piezoelectric CCLD sensor (model 4507-B-005 from the Brüel \& Kjaer company) and the external sound card (model E-MU Tracker Pre from the Creative company) connected to the Lenovo Ideapad laptop, with the Adobe Audition software controlled by the Windows 7 operating system. This way the portable system was crated, easily movable to the desired locations, responsible for recording the sound from the sensor and saving it to the binary wavfile. The applied sensor is a cheap solution, yet adjusted to accurately measure sound carried in the wood. It is simpler than any other device with comparable accuracy, such as the laser vibrometry or piezoelectric sensor. It is also easily attached to the piece of wood (glued to the surface), without any intrusive technique (such as drilling holes).

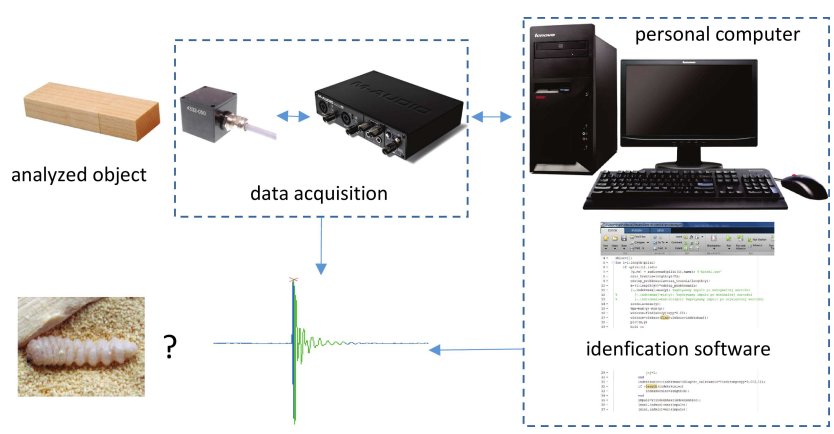

Fig. 2. Scheme of the wood boring insects detection system.

Next, its content may be processed by the identification module, operating on the same computer in the off-line mode. The identification process consists of two consecutive steps. The first one is the detection of the sound that might be caused by the larva. Subsequently, the identification procedure is executed. Its resolution and accuracy depends on the desired effect. In the simplest case the aim of the module operation is determining the presence of the insect in the analyzed wooden construction. In the most desirable configuration, various species of the vermin would be discovered. The advantages of the proposed approach include the low cost (only the single sensor is required) and the flexibility - the identification method may be selected from the broad area of algorithms. The drawbacks include the difficulty of detecting and identifying the insect species, especially in the noisy environment, as will be discussed below.

Even in the simplest application, the problem of the proposed scheme is the existence of other sources of vibrations, recorded by the accelerometer. Such sources as the human voice, working machinery, accidental interaction with the surrounding environment may be the serious obstacle for the proper and accurate woodworm detection. Such sounds may be treated as the background noise, which should be suppressed if possible. The obvious solution to this problem is using the isolated laboratory premises, where all unwanted sound sources would be eliminated. Unfortunately, such isolated environment can't be used in many situations, for instance, when the analysis is to be performed in the wooden building. Although the laboratory recordings are still useful (for instance, while creating the training vectors for the classifiers), the methodology must be tested in terms of distinguishing between the larvae and non-insect objects. It is important to determine which sounds are recorded by the accelerometer and what are their characteristics, usable for separating the particular categories of sounds. The problem of extracting the larva sound from the background noise was considered in (SCHOFIELD, 2011). The fractal dimension analysis was used there as the detection module. It required setting the threshold above which the dimension represents the sound, and not the background noise. As the alternative, the sound energy can be analyzed, similarly requiring the threshold, above which the signal is considered a candidate for the larva identification. In our experiments it was assumed that every sound above the threshold must be processed, which allows for using the latter approach, requiring smaller amount of computations.

Two configurations of recorded sounds are possible to cause the problem for the system:

- The sound recorded from the single source, either the larva or another object (such as the human being or the electrical device). In this case, the system must only make decision whether the vibration is caused by the insect or not. This scheme requires the binary classification, separating insectoriginated sounds from all other.

- The sound is the superposition of vibrations coming from various sources at the same time. This is the more demanding situation, as multiple combinations of sounds require the more detailed research, both regarding the useful signal features and the particular classifier.

To solve both problems, three stages of operation should be prepared. The first one is the initial assessment of the created system where the background sounds are selected randomly. The second stage involves selecting the background sounds as close to the larva-originated as possible. Finally, the ability 
to distinguish between the sounds of old house borer species and the larvae of wood boring insects should be checked. In the presented research the first step is verified.

Although detection of the larva in the isolated environment is relatively simple (as long as the insect makes sounds while dwelling in the wood), its identification may be difficult. This is because the sound made by the specimen depends on the series of factors, which must be considered during the design of the automated classifier:

- The activity of the larva depends on the temperature and humidity. It is important to know the behaviour of the specific insect species and prepare the recording conditions to increase the chance of detecting the larva.

- The recorded sounds will differ for the same species, depending on the age and size of the larva.

- The type of the wood, in which the insect feeds is also important. The sound produced by the larva is the sound of breaking fibers. Their condition and structure influences the particular sound pattern. For instance, the behaviour of the wet and dry matter gives quite different results, although the same insect lives inside.

\section{Artificial intelligence classification method}

The presented scheme is assumed to exploit the automated classification method. The AI is currently the popular approach to solve similar problems. The application of the particular algorithm to the task requires implementing the following operations (Fig. 3):

- Training on the available data set. This process consists in extracting knowledge from the available vectors of features and storing it in the form characteristic for the particular algorithm. This operation belongs to the machine learning domain (Mitchell, 1997).

- Exploitation of the previously extracted knowledge to make decisions about the presence or absence of the wood boring insects in the analyzed piece of wood. This way the applied method works as the expert system.

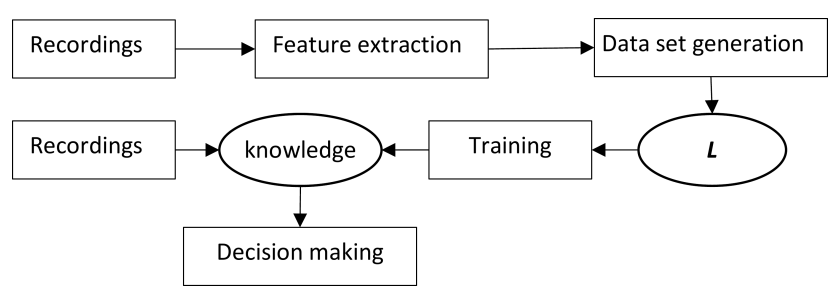

Fig. 3. AI-based wood boring insect detection scheme.

The AI-based decision making is performed based on the vector of features, extracted from the sound waveform. Their selection has the key importance for the accurate classification of objects. This requires the analysis by the human expert, having knowledge about the nature of the signal. The first step in creating data for training the classifier involves listening to the recorded samples, trying to determine, which sound is made by the worm, contrary to the background noise.

Preparation of the samples is a difficult task, consisting in breeding the larvae in the specified pieces of wood. Because such insects spend most of their lives in the larva form, this process is time consuming. The specimens must be held in the proper conditions (temperature and humidity), breeding for a couple of months. After they reach the adolescence, the recording trials may commence. For the presented research, two groups of objects must be recorded: larvae and other sources (caused deliberately during the experiment, such as scratching the surface of the wooden piece, talking, etc.). This way the information about the characteristics of the selected sounds is collected. They are all required to determine, which sounds are the most difficult to distinguish from the insectoriginated. At this stage, the recordings should be made in the isolated environments (such as the anechoic chamber), where occurrence of all sounds is controlled during the experiment. After recording the behaviour of the larva, every piece of wood is examined to see if the insect is still alive and what is its condition (estimated age, weight, or length). This information can be used to correlate the nature and intensity of sounds with the state of the larva. Next, the human expert is able to select the most representative sounds made by larvae during feeding to extract the features (symptoms) further used to distinguish between the insect and other sound source, or identify the particular species.

Selection of features for the analysis is the second step prior to the training. The vector of symptoms $\mathbf{s}=\left\{s_{1}, \ldots, s_{m}\right\}$ extracted from the waveform $\mathbf{y}(t)$ should be enough to produce the function $f(\mathbf{s})$ mapping them to the discrete category $c \in Z$, describing the insect or the background noise in the simplest case (the binary classification scheme, where $c=\{0,1\}$ ) or distinguishing between the particular species of insects (multiple classification scheme, where $|c|>2$ ). Depending on the application, various domains should be considered. The sound caused by larvae is the waveform similar to the impulse response of the linear system (Fig. 4) - (EverEst, 2000). This means the high pitch at the very beginning, caused by breaking the wooden fiber, quickly decreases to the background level. In the binary classification scheme, when only one sound source is active at the moment, the time domain should be enough to extract the useful knowledge. When multiple sources overlap, the additional domains may be required, representing the signal in the frequency or the scale domains, calling for 

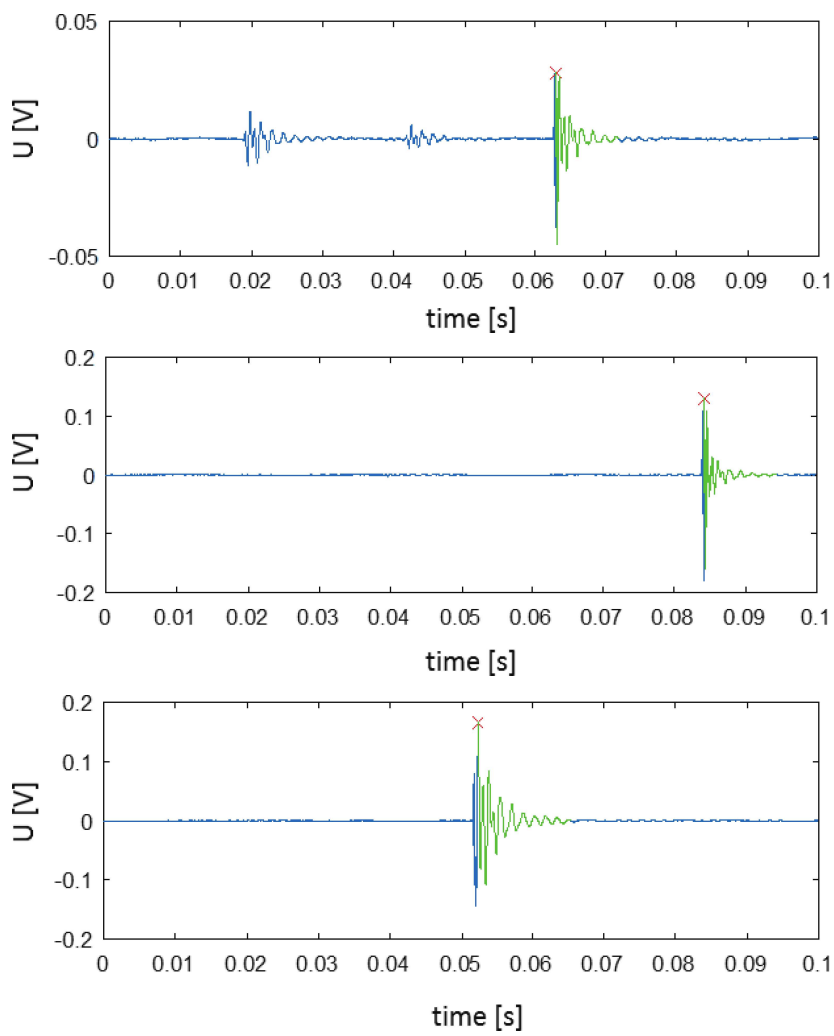

Fig. 4. Recorded signals of the wood borer insect cracking the fiber (on the example of the larvae of the old house borer).

the Fourier or Wavelet transforms. In (SCHOFIELD, 2011) the frequency domain was considered too computationally costly to be used online. Today it is no longer the case, therefore both time features and frequency characteristics may be exploited. In the first stage of the presented work, the time domain features are enough to correctly identify the analyzed sounds. Although the sound may be processed to search for the interesting features (for instance, using the relational trees - (SCHOFIELD, 2011)), the most accurate way is to select features manually and then compress them to the most important characteristics (for instance, by using the correlation analysis). This was our solution. As can be observed in Fig. 4, the impulses caused by the cracking wood are of various amplitudes and times of fading away. Their common trait is the intensity of the waveform decrease. The key to describe the signal is then finding the moment where the impulse starts and ends. The part of the signal identified by the green color is considered the impulse duration.

The example of the sounds coming from other sources are in Fig. 5. In most cases the waveforms are easy to distinguish from the larva-originated impulses. Duration of the considered signal is much longer here, and fading has different character (the subsequent extreme values are more numerous or located in the greater distance from each other). In some cases, the waveforms are similar to the larva-originated im-
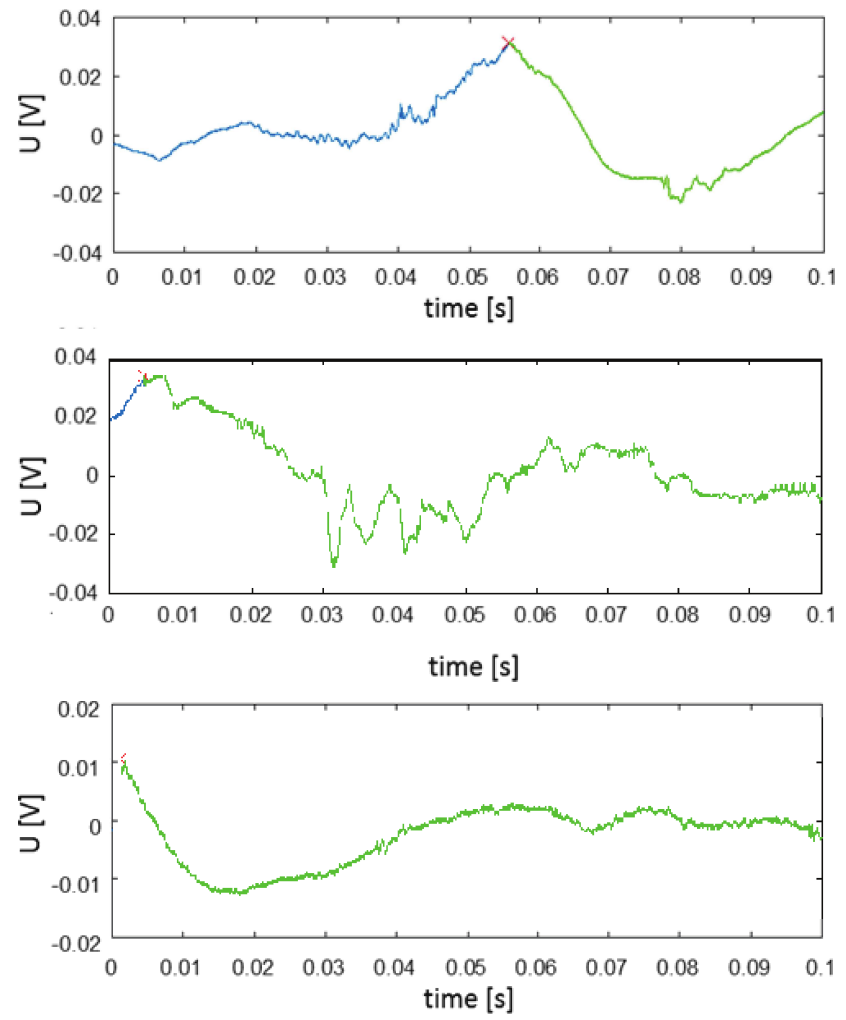

Fig. 5. Recorded signals coming from other sources.

pulses, which may cause difficulties in distinguishing between these two categories.

Based on the analysis of the positively confirmed sounds (i.e. indeed made by the larva), the following time domain features were extracted from the waveforms (Fig. 6):

a) The difference $t_{d r}$ between the maximum and minimum value of the voltage within the impulse

$$
t_{d r}=\left|t\left(\max \left(\mathbf{y}_{i}\right)\right)-t\left(\min \left(\mathbf{y}_{i}\right)\right)\right|,
$$

where $\mathbf{y}_{i}$ is the part of the waveform identified as the impulse (indicated with the green colour in Fig. 4 and Fig. 5). The impulse is extracted from the waveform starting at the high pitch and lasting till the power of the impulse fades to the noise level. The method for detecting the start instance $t_{b}$ of the impulse consists in searching for the maximum or the minimum value in the waveform, or the one of these two that is greater (by comparing absolute values):

$$
t_{b}=\left\{\begin{array}{l}
t(\max (\mathbf{y})) \\
t(\min (\mathbf{y})), \\
t(\max (\operatorname{abs}(\mathbf{y})))
\end{array}\right.
$$

All three methods we tested during the experiments.

The end of the impulse is considered the time instance, for which the power of every cumulative 


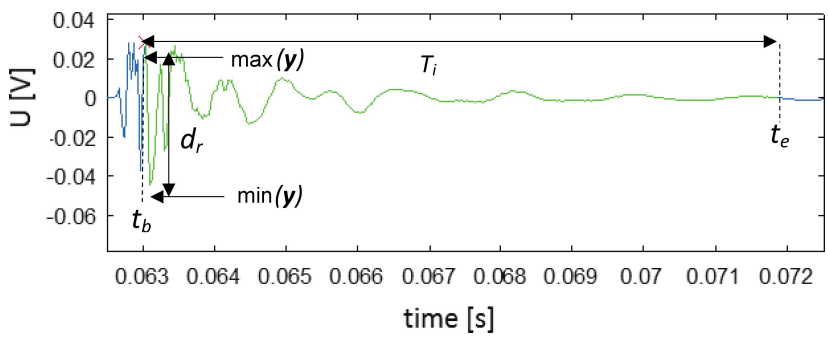

Fig. 6. Illustration of the features extraction from the waveform.

set of samples goes below the certain threshold $\theta$, here equal to 0.01 of the dynamic range in the processed fragment of the waveform:

$$
t_{e}=t: \sum_{t=t_{i}}^{t_{i+n}} \mathbf{y}(t)<\theta \cdot d_{r}
$$

b) Maximum and minimum value of the impulse waveform $\mathbf{y}_{i}$ amplitude.

c) Dynamic range $d_{r}$ in the impulse waveform

$$
d_{r}=\max \left(\mathbf{y}_{i}\right)-\min \left(\mathbf{y}_{i}\right) .
$$

d) Impulse duration for the previously defined border values $t_{b}$ and $t_{e}(2)$ and (3):

$$
T_{i}=t_{e}-t_{b}
$$

e) Root mean square value of the pulse.

In Table 1 the numerical values of features corresponding to Fig. 6 are presented. Note that the sound level varies significantly between samples. This is the main source of the problem in detecting sounds in the noisy environment, as some sounds may be too weak to trigger the identification procedure outside the isolated chamber.

Table 1. Examples of features extracted from the detected impulse.

\begin{tabular}{|c|c|c|c|c|c|c|}
\hline $\begin{array}{c}t_{d r} \\
{[\mathrm{~ms}]}\end{array}$ & $\begin{array}{c}\max (\mathbf{y}) \\
{[\mathrm{mV}]}\end{array}$ & $\begin{array}{c}\min (\mathbf{y}) \\
{[\mathrm{mV}]}\end{array}$ & $\begin{array}{c}d_{r} \\
{[\mathrm{mV}]}\end{array}$ & $\begin{array}{c}T_{i} \\
{[\mathrm{~ms}]}\end{array}$ & $\begin{array}{c}\mathrm{RMS} \\
{[\mathrm{V}]}\end{array}$ & $c$ \\
\hline 0.068 & 100 & -117 & 218 & 9.52 & 3.15 & 1 \\
\hline 0.341 & 26.7 & -44.7 & 71.4 & 10.2 & 1.45 & 1 \\
\hline-1.024 & 34.3 & -19.7 & 54.1 & 9.82 & 1.86 & 0 \\
\hline
\end{tabular}

Because all vectors are created for waveforms where the source of the sound is known, data sets consist of the labeled examples $\mathbf{e}=\{\mathbf{s}, c\}$, where $c$ is the category of the example. In the binary case two values are used (larva or the background noise), in multiple categories identification particular species of the insects can be detected. Such a set $\mathbf{L}$ is the input for training of the classifier, which analyzes examples and finds relations between the particular symptoms and the categories

$$
\mathbf{L}=\left[\begin{array}{cccc}
s_{11} & \cdots & s_{1 m} & c_{1} \\
\vdots & \ddots & \vdots & \vdots \\
s_{n 1} & \cdots & s_{n m} & c_{n}
\end{array}\right]
$$

\section{Support Vector Machines}

The Support Vector Machines (SVM) are currently one of the most popular and powerful classifiers in technical (KUREK, OsowsKI, 2010) and environmental (CHEHADE et al., 2009) sciences. They have proven their usefulness in multiple applications, especially in the presence of uncertainty conditions, such as the background noise and limited accuracy of the measurement equipment. Therefore, they are currently preferred over the always popular ANN, also used in the acoustic analysis of wood boring insects (SCHOFIELD, 2011). The single SVM for classification produces the binary output, i.e. two values $\{-1,1\}$. The classification of multiple species of insects requires combining binary classifiers into configurations, such as One-vsAll or Multiple-Output-Coding (SHEN et al., 2010). In the presented research only the single binary classifier is required, its aim is isolate the larva from the background noise. The SVM module is the black box (Fig. 7), having the vector of five or six features (depending on whether the difference between the maximum and minimum impulse value is considered) on the input and one output $d$, responsible for making decisions. Here $d$ stands for the binary decision value ("larva" vs "environmental sound") and $g$ is the weighted sum of inputs $s$ transformed by the kernel function $K$ :

$$
g(\mathbf{s})=\sum_{i=1}^{m} K\left(\mathbf{s}_{i}, \mathbf{s}_{j}\right) \cdot w_{i} .
$$

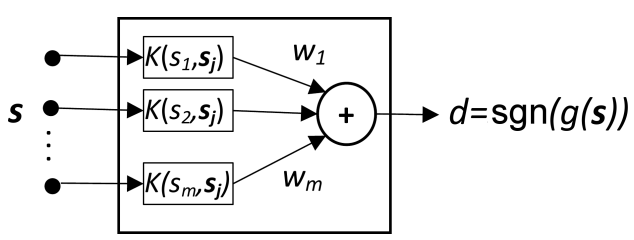

Fig. 7. Structure of the binary SVM classifier (BILSKI, 2013).

Selection of the kernel function and its parameters is one of the most important operations during the SVM training (Patle, Chouhan, 2013). Currently the Radial Basis Function (RBF) is considered the most effective. Its parameter is the width $\gamma$ of the bell function curve around the mean value. During the research, also polynomial (with its degree $p$ as the parameter) and linear (no parameters) kernels have been utilized

$$
\begin{aligned}
K_{\mathrm{RBF}}\left(\mathbf{s}_{i}, \mathbf{s}_{j}\right) & =\exp \left(-\gamma \cdot\left\|\mathbf{s}_{i}-\mathbf{s}_{j}\right\|^{2}\right) \\
K_{\mathrm{POLY}}\left(\mathbf{s}_{i}, \mathbf{s}_{j}\right) & =\left(\mathbf{s}_{i}^{T} \cdot \mathbf{s}_{j}+c\right)^{p}
\end{aligned}
$$

Here $c$ is the free parameter defining the compromise between importance of the high and low order terms in the polynomial. In the presented research it was set to 1 . 
The scheme of the SVM application to the described problem is presenting the set $\mathbf{L}$ to the module during the training for the predefined kernel (with its parameters defined). Then, the efficiency of the module is verified on the tested data set $\mathbf{T}$ of the same form as $\mathbf{L}$, but containing different examples. To generalize the evaluation, the training-testing procedure was repeated for various configurations of $\mathbf{L}$ and $\mathbf{T}$ using the Repeated Random Sub-Sampling Cross-Validation (RRSSCV) (DE LACERDA et al., 2002). The presented identification module was written in the Matlab environment, using the SVM Toolbox.

\section{Experimental results}

The presented experiments were performed on the data coming from recordings of the old house borer, which is relatively easy to breed, therefore the Authors were able to prepare multiple examples describing its behaviour. All used samples were bred in the same type of wood (used to construct the furniture). The aim of the procedure was to check the ability of the single accelerometer DAQ-based module to distinguish between the larva and the background noise. Sixteen pieces of wood with insects inside were processed. Every piece was analyzed for 45 minutes, during which the sensor was attached to the wooden block and the sound recorded to the file. Each recording was then analyzed by the human expert, who was able to extract the larva activity and identify impulses related to cracking the fiber structure. This way 127 insect-originated impulses were proposed to create the data set. Additionally, 63 samples were prepared with sounds coming from other sources (i.e. human voice, walking around the premise, scratching the surface of the sample or the table on which it was positioned). This way the group of background signals of varying resemblance with the larva-originated ones was created, giving overall 190 examples labeled with categories " 1 " (for the larva) and " 0 " for the background noise. This is the larger data set than used in most of experiments, such as (SCHOFIELD, 2011).

Examples belonging to each category were then randomly divided into two groups in the ratio 3:7 and assigned to the testing and training set, respectively. The process was repeated fifteen times to obtain different sets for training and evaluating the classifier. The size of $\mathbf{L}$ was 109 examples, while of $\mathbf{T}-81$ (containing examples belonging to both categories).

The average classification outcomes are presented in Table 2 for various configurations of SVM - i.e. the kernel function and its parameters. The selected accuracy measure uses the sample error $E_{s}(\mathbf{T})$, i.e. the relative number of incorrectly classified examples

$$
\operatorname{acc}=1-E_{s}(\mathbf{T})=1-\frac{|\mathbf{e}: c(\mathbf{s}) \neq d(\mathbf{s})|}{|\mathbf{T}|} .
$$

Table 2. Classification results [\%] of the SVM module for various kernels and methods of detecting the beginning of the impulse.

\begin{tabular}{|c|c|c|c|}
\hline \multirow{2}{*}{ Kernel } & \multicolumn{3}{|c|}{$t_{b}$} \\
\cline { 2 - 4 } & abs & $\max$ & $\min$ \\
\hline $\operatorname{RBF}(\gamma=1)$ & 88.67 & 85.06 & 86.33 \\
\hline Polynomial $(p=3)$ & 88.17 & 87.13 & 86.56 \\
\hline Linear & 86.67 & 84.95 & 84.59 \\
\hline
\end{tabular}

The RBF kernel confirms its greatest usefulness in the classification of objects. The polynomial kernel has the similar accuracy. The main problem in both cases is adjustment of the kernel parameter to maximize the classification accuracy. The data sets are of the moderate difficulty, as the linear kernel also achieves the high accuracy. The beginning of the impulse detection based on the largest absolute value in the waveform provides the greatest identification accuracy, although the remaining methods also give acceptable outcomes.

The process of the kernel parameter optimization is presented in Figs. 8 and 9 (for the RBF and polynomial functions, respectively). The width of the RBF must be above the threshold value, then reaches the stable range, for which differences in the classification accuracy are of the secondary importance. On the other hand, the polynomial degree should not be too large, ensuring the proper generalization ability. Too complex function results in the significant decrease of the SVM accuracy.

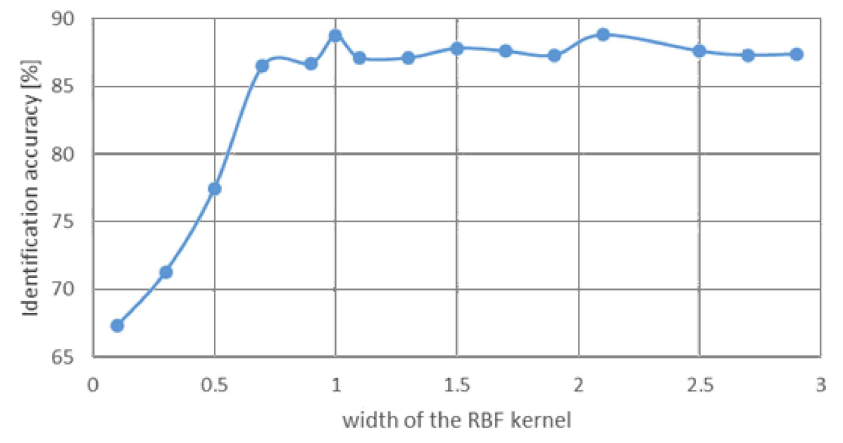

Fig. 8. Optimization process of the RBF kernel parameter.

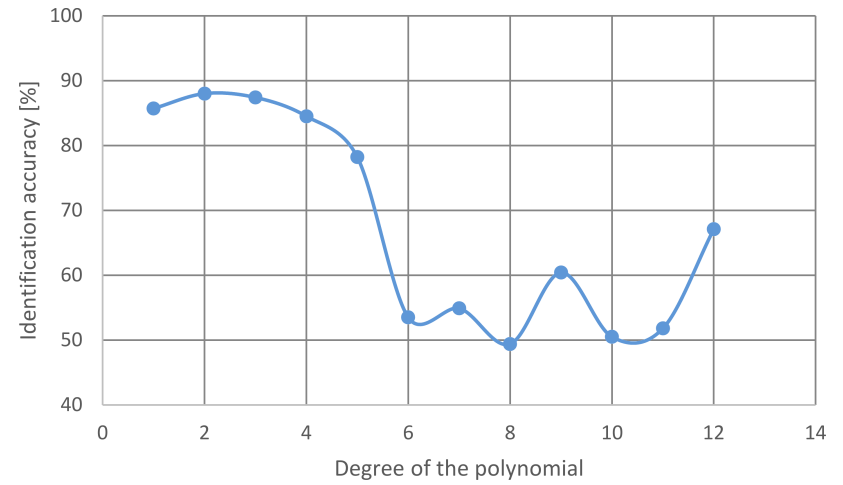

Fig. 9. Optimization process of the polynomial kernel parameter. 
The analysis of which examples are difficult to distinguish for the SVM classifier shows that most of the errors are made by the modules for the category "0" (background noise), sometimes mistaken by the impulse made by the larva. For the binary classification four outcomes are defined: True Positive (TP), i.e. the number of examples with correctly detected larvae, True Negative (TN), i.e. the number of examples with correctly detected the background noise, False Positive (FP), which is the number of false alarms (incorrectly detected larvae) and False Negative (FN), i.e. the number of incorrectly detected background noise. Detailed outcomes for all kernels processing the data where the beginning of the impulse is the maximum absolute value are in Tables 3-5 (the number of all examples in the set is 58). The additional quality parameters are sensitivity (11) and specificity (12), where $P$ is the number of actual larva examples and $N$ is the number of actual background noise examples in the set. Their values are given near the corresponding tables

$$
\begin{aligned}
& \text { sens }=\frac{\mathrm{TP}}{P} \cdot 100 \%, \\
& \text { spec }=\frac{\mathrm{TN}}{N} \cdot 100 \% .
\end{aligned}
$$

Table 3. Detailed results of the classification using the RBF kernel (sens $=94.87 \%$, spec $=73.68 \%$ ).

\begin{tabular}{|c|c|c|}
\hline \multirow{2}{*}{ Actual } & \multicolumn{2}{|c|}{ Predicted } \\
\cline { 2 - 3 } & 0 & 1 \\
\hline 0 & $\mathrm{TN}=14$ & $\mathrm{FP}=5$ \\
\hline 1 & $\mathrm{FN}=2$ & $\mathrm{TP}=37$ \\
\hline
\end{tabular}

Table 4. Detailed results of the classification using the polynomial kernel ( sens $=92.30 \%$, spec $=63.15 \%$ ).

\begin{tabular}{|c|c|c|}
\hline \multirow{2}{*}{ Actual } & \multicolumn{2}{|c|}{ Predicted } \\
\cline { 2 - 3 } & 0 & 1 \\
\hline 0 & $\mathrm{TN}=12$ & $\mathrm{FP}=7$ \\
\hline 1 & $\mathrm{FN}=3$ & $\mathrm{TP}=36$ \\
\hline
\end{tabular}

Table 5. Detailed results of the classification using the linear kernel $($ sens $=89.74 \%$, spec $=78.94 \%)$.

\begin{tabular}{|c|c|c|}
\hline \multirow{2}{*}{ Actual } & \multicolumn{2}{|c|}{ Predicted } \\
\cline { 2 - 3 } & 0 & 1 \\
\hline 0 & $\mathrm{TN}=15$ & $\mathrm{FP}=4$ \\
\hline 1 & $\mathrm{FN}=4$ & $\mathrm{TP}=35$ \\
\hline
\end{tabular}

In all cases the number of incorrectly detected background noise instances is equal or greater than the number of incorrectly detected larvae samples. The larva samples are detected in most cases by all kernels. The main problems for the SVM classifier are waveforms with the background noise similar to the actual impulse caused by the larva. Such are the sounds of scratching the surface with the finger or the sharp object. On the other hand, the human voice is either absent in the recording or easily distinguishable from the behaviour of the larva.

The high accuracy of the detection is caused by the proper selection of features allowing to distinguish between two categories and the good generalization ability of the SVM. In should be noted that the background noise samples were prepared without the sounds considered the most similar to the impulses caused by the larvae - such as hitting the table, on which the wood piece is located with the key. Such examples may require additional features to incorporate into the detection system. Additionally, separating the particular insect species from each other may be another challenge, as sounds created by various specimens may also be similar. This includes the difficulty in identifying the particular insect species living in various types of wood, also presenting different conditions. For instance, the larva feeding on the furniture wood sounds different from the specimen living in the tree. Such facts must be considered in the incoming investigations.

\section{Conclusions}

The paper presented the application of the simple and low-cost system for detecting the wood boring insects presence in the wooden constructions. Its current accuracy, close to 90 percent, is satisfactory at the first step of the presented works. The advantages include the low cost (covering only the single accelerometer and the sound card), portability (thanks to the usage of the laptop computer) and flexibility (as the system after the calibration can be used to identify also other species of larvae).

The decision making part based on the binary SVM classifier, proved its usefulness, also in the uncertainty conditions, which is widely considered a problem of the traditional ANN. It was also confirmed that the selection of the SVM configuration is essential to maximize the system's efficiency. The feature vectors used by the classifier (belonging to the time domain) are enough for such a simple task, where only one source of the sound is possible at a time.

The future extension of the proposed method includes adding frequency domain features (such as the spectral samples) to the vector, which would allow for distinguishing between various sources of the signal operating simultaneously. Also, other AI-based classifiers (such as the fuzzy logic) should be tested. Finally, introduction of more species of insects would greatly increase the applicability of the approach. Usage of SVM for such a complex task would require the strategy for the combination of the binary classifiers, not only detecting the larva presence, but also suggesting 
the species. This will also require preparation of large data sets describing the behaviour of new insects.

\section{References}

1. BiLsKi P. (2013), Artificial intelligence methods in the diagnostics of analog systems, Oficyna Wydawnicza Politechniki Warszawskiej, Warsaw.

2. Chehade N.H., Boureau J.G., Vidal C., ZeruBIA J. (2009), Multi-class SVM for forestry classification, Proceedings of the 16th IEEE International Conference on Image Processing (ICIP), 1673-1676.

3. Chiappini E., Nicolialdini R. (2011), Morphological and physiological adaptations of wood-boring beetle larvae in timber, Journal of Entomological and Acarological Research, Ser II, 43, 2, 47-59.

4. Everest F.A. (2000), Master Handbook of Acoustics, McGraw-Hill, New York.

5. FARR I. (2007), Automated Bioacoustic Identification of Statutory Quarantined Insect Pests, PhD thesis, University of York.

6. Fiala P., Friedl M., Cap M., Konas P., Smira P., Naswettrova A. (2014), Non Destructive Method for Detection Wood-destroying Insects, PIERS Proceedings, Guangzhou, China, 1642-1646.

7. Fleming M.R., Bhardwaj M.C., Janowiak J.J., Shield J.E., Roy R., Agrawal D.K., Bauer L.S., Miller D.L., Hoover K. (2005), Noncontact ultrasound detection of exotic insects in wood packing materials, Forest Products Journal, 55, 6, 33-37.

8. Hetzroni A., Soroker V., Cohen Y. (2016), Toward practical acoustic red palm weevil detection, Computers and Electronics in Agriculture, 124, 100-106.

9. Hoffmann N., Schröder T., Schlüter F., Meinlschmidt P. (2013), Potential of infrared thermography to detect insect stages and defects in young trees, Journal Für Kulturpflanzen, 65, 9, 337-346.

10. Kerner G., Thiele H., Unger W. (1980), Secure and robust detection of the woodworm larvae [in German: Gesicherte und zestörungsfreie Ortung der Larven holzzestörender Insekten im Holz], Holztechnologie 21, 131-137.

11. Krajewski A., Witomski P. (2005), Videoendoscopy as the method dor the asessing the state of the wooden constructons' monuments [in Polish: Videoendoskopia jako metoda oceny stanu drewnianych konstrukcji w zabytkach], Ochrona Zabytków, 4, 105-108.

12. Krajewski A., Witomski P., Bobiński P., WóJCIK A., Nowakowska M. (2012), An attempt to detect fully-grown house longhorn beetle larvae in coniferous wood based on electroacoustic signals, Drewno. Prace naukowe. Doniesienia. Komunikaty, 55, 108, 5-15.

13. Kurek J., Osowski S. (2010), Support vector machine for fault diagnosis of the broken rotor bars of squirrelcage induction motor, Neural Comput \& Applic, 19, $557-564$
14. De Lacerda E.G.M., De Carvalho A.C.P.L.F., LuDERMIR T.B. (2002), A study of cross-validation and bootstrap as objective functions for genetic algorithms, Proceedings of the VII Brazilian Symposium on Neural Networks, 118-123.

15. Lemaster R.I., Beall F.C., Lewis V.R. (1997), Detection of termites with acoustic emission, Forest Product Journal, 47, 2, 75-79.

16. Mankin R.W., Weaver D.K., Grieshop M., LarSON B., Morril W.L. (2004), Acoustic system for insect detection in plant stems: comparisons of Cephus cinctus in wheat and Metamasius callizona in bromeliads, Journal of Agricultural and Urban Entomology, 21, 239-248.

17. Mikolajska A., Walczak M., Kaszowska Z., URBAŃCZYK-ZAWADZKA M., BANYŚ R.P. (2012), Xray techniques in the investigation of a Gothic sculpture: The risen Christ, Nukleonika, 57, 4, 627-631.

18. Mitchell T.M. (1997), Machine Learning, McGrawHill, New York.

19. Osterloh K.R.S., Nusser A. (2014) X-ray and neutron radiological methods to support the conservation of wooden artworks soaked with a polluting impregnant 'Carbolineum', Proceedings of the 11th European Conference on Non-Destructive Testing (ECNDT 2014), October 6-10, 2014, Prague, Czech Republic.

20. Patle A., Chouhan D.S. (2013), SVM kernel functions for classification, International Conference on Advances in Technology and Engineering (ICATE), 19 .

21. Pence R.J., Magasin S.J., Nordberg R.G. (1954), Detecting wood-boring insects electronic device developed as aid in locating insects destructive to timber and wood products, California Agriculture, 5.

22. Schofield J. (2011), Real-time Acoustic Identification of Invasive Wood-boring Beetles, $\mathrm{PhD}$ thesis, University of York.

23. Shen Y., LiU G., LiU H. (2010), Classification method of power quality disturbances based on RVM, Proceedings of the 8th World Congress on Intelligent Control and Automation (WCICA), 6130-6135.

24. Stirr S.A., Waddle J.R. (1999), Use of CT in Detection of Internal Damage and Repair and Determination of Authenticity in High-Quality Bowed Stringed Instruments, RadioGraphics, 19, 639-646.

25. Stusek P., Pohleven F., Capl D. (2000), Detection of wood boring insects by measurement of oxygen consumption, International Biodeterioration and Biodegradation, 40, 293-298.

26. Wood-boring Beetles of Structures, Texas A\&M AgriLife Extension, 12/11, 1-5.

27. Zorović M., CoKL A. (2015), Laser vibrometry as a diagnostic tool for detecting wood-boring beetle larvae, Journal of Pest Science, 88, 107-112. 